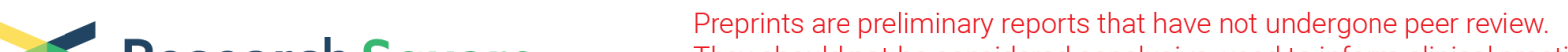 $\begin{array}{ll}\text { Research Square } & \text { They should not be considered conclusive, used to inform clinical practice, } \\ \text { or referenced by the media as validated information. }\end{array}$
}

\section{Spontaneous malignant transformation of ovarian surface epithelial cells correlates with EMT alteration and stemness acquisition}

Bingyan Li ( $\boldsymbol{\sim}$ bingyanli@suda.edu.cn )

Soochow University https://orcid.org/0000-0002-0404-7709

Hemei Zhang

Wenzhou Center for Disease Control and Prevention

Yi Guo

Jiangpu Community Hospital

Lizhi Liu

Sun Yat-sen University Cancer Center

\section{Yongfeng Hou}

State key laboratory of Cardiovascular Disease, Fuwai Hospital, Beijing, Chian

\section{Ping Wang}

Fujian Key labortary of Transplate Biology, Fuzhou General Hospital, Fuzhou, China 350025

\section{Fei Jiang}

School of Publich Health, Soochow University, Suzhou, China

\section{Zengli Zhang}

School of Pulbich Health, Soochow University, Suzhou, China

\section{Tom K Hei}

Department of Radiation Oncology, College of Physicians and Surgeons, Columbia University, New York, NY USA

\section{Research article}

Keywords: epithelial-mesenchymal transition, cancer stem-like cells, ovarian surface epithelial, malignant transformation, epithelial ovarian carcinomas

Posted Date: October 17th, 2019

DOI: https://doi.org/10.21203/rs.2.15828/v2

License: (c) (i) This work is licensed under a Creative Commons Attribution 4.0 International License.

Read Full License 


\section{Abstract}

Background In order to complete the physiological function of postovulatory repair during repeated ovulation, the ovarian surface epithelium (OSE) not only has to undergo epithelial-mesenchymal transition (EMT), but also possess the properties of somatic stem-like cells. However, there is no evidence to indicate that both EMT alteration and stemness acquisition are linked to epithelial ovarian carcinomas.Methods In this study, we established a cell model of spontaneous oncogenic transformation of mouse OSE (MOSE). The cell proliferation was assessed using clonogenic survival and soft agar. The self-renewal of cancer stem-like cells (CSCs) was determined by spheroid culture. CD44 + /CD117 + cells were analyzed using flow cytometer. The PCR array was used to determine the EMT-related mRNA level. Expression of pan-keratin, vimentin, E-cadherin, Snail1 and Slug were detected using western-blotting and immunofluorescences, respectively. The tumorigenesis were monitored by limiting dilution assay in vitro and in vivo .Results Based on morphological change, chromosomal number and proliferating ability, we defined three sequential stages of transformed cells as early, intermediate and late MOSE cells, respectively. We found that MOSE cells had dual characteristics of not only epithelial but mesenchymal nature as well. Over time, MOSE cells spontaneously developed characteristics of malignant cells and generated tumor nodules expressing both Pan-keratin and Vimentin. Furthermore, we found that the neoplastic transformation of MOSE was accompanied by continuous EMT-inducing signals including Snail1 and Slug. Concurrently, the increase of CD44 + /CD117 + cells and their self-renewal ability were associated with the progression of spontaneous neoplastic transformation of MOSE cells in vitro. Conclusion These results indicated that both EMT alteration and stemness acquisition were closely correlated with the spontaneous malignant progression of MOSE cells. Our findings provide new insights into the future to combat epithelial ovarian carcinomas.

\section{Background}

Ovarian cancer is a deadly gynecologic malignancy with a five-year survival rate of less than $30 \%$ [1]. The poor prognosis is largely due to difficulties in its early diagnosis and the high rate of relapse as a result of chemoresistance. Epithelial ovarian carcinomas (EOC) account for $90 \%$ of all ovarian malignancies [2] and comprise multiple subtypes [3]. The early events of EOC development are poorly understood and the exact initiating cell population remains to be defined.

Ovarian surface epithelium (OSE), a single layer of squamous or cuboidal cells, plays physiological roles in both follicular rupture and subsequent ovarian remodeling by altering the motility and proliferation response necessary for extracellular matrix remodeling. OSE can undergo proliferative repair, and its normal regulation may contribute to the pathogenesis of EOC [4]. In addition, OSE has been postulated as the source of EOC base on pathological observations and experimental approaches [5-8].

The capacity of OSE that undergoes epithelial-mesenchymal transition (EMT) in response to postovulatory stimuli has been proposed to confer advantage to the postovulatory repair of the epithelia cells [9]. Hence, EMT is a part of normal OSE physiology and failure in this process may be the reasons of 
ovarian cancer initiation and metastasis. Additionally, the cyclic pattern of repeated disruption and repair with complex remodeling associated with ovulation leads one to intuit the existence of a population of somatic stem cells that would be responsible for these processes. OSE with some stem-cell properties have been identified previously based on their slow proliferation [10]. In addition, OSE at the junction area contains cancer-prone stem cell niche [11]. Moreover, accumulating evidence supports that EMT and cancer stem cells (CSCs) play critical roles in the development of chemoresistance, tumor relapse and metastasis in ovarian cancer patients [12-14].

In order to complete the physiological function of postovulatory repair during repeated ovulation, OSE not only has to undergo EMT, but also possess the properties of somatic stem-like cells. The current literatures on the existence of somatic stem cells and EMT-associated events in normal OSE have discussed its implication for normal ovarian function as well as neoplastic phenotypes [9, 15-18]. Distinctly, OSE possesses extraordinary cellular proliferative capacity without reaching crisis phase [19], while other adult epithelial cells undergo a restricted number of population doublings before senescence in vitro. Several experimental models have recently been developed to study the transformation of OSE, including genetically modified mouse models and manipulation of cultured human OSE cells [20-28]. In addition, the transcriptional profile of ovarian carcinomas was investigated $[9,16,18]$. There is, however, no evidence indicating that EMT alteration and stemness acquisition are involved in the spontaneous transformation of OSE cells.

The isolation and culture of human and mouse ovarian surface epithelium have been described [24, 27 , 28]. Therefore, taking advantage of the spontaneous transforming of OSE cells, we established a stepwise neoplastic transformation model of these cells from a premalignant phenotype to the malignant one. We also demonstrated that the alteration of EMT and acquisition of stem-like properties were associated with the neoplastic process of mouse OSE cells.

\section{Methods}

\section{Cell isolation and culture}

Mouse ovarian surface epithelial (MOSE) cells were isolated as described by Roby and Paul et al [1-3], and modified by us. Briefly, ovaries from female C57BL/ 6 mice (Experimental Animal Center for Soochow University, ) were resected and, were incubated for 20 min in Dulbecco's Modified Eagle's Medium/Nutrient Mixture F-12 (DMEM/F12, Invitrogen, Carlsbad, CA) supplemented with 0.25\% trypsin (Invitrogen, Carlsbad, CA). Single cells and clumps of MOSE were collected, resuspended in MOSE cell growth medium, and seeded onto $35 \mathrm{~mm}$ dishes in a humidified atmosphere of $5 \% \mathrm{CO}_{2}$ at $37^{\circ} \mathrm{C}$. The MOSE cell growth medium consisted of DMEM/F12 medium supplemented with $5 \%$ fetal bovine serum (FBS), 10 $\mathrm{ng} / \mathrm{ml}$ mouse epidermal growth factor (mEGF), $100 \mathrm{U} / \mathrm{mL}$ penicillin and $100 \mu \mathrm{g} / \mathrm{mL}$ streptomycin (Beyotime Biotechnology, shanghai, China), 1\% insulin-transferrin-selenium (Invitrogen, Carlsbad, CA). During early passages of cells (pass 1 through 20), medium was changed with the mixed medium containing a 1:1 of the fresh growth medium and culture supernatant. Intermediated (pass 21-90) and 
late passage (great than 90) cells were routinely passaged. This animal experiment was performed according to the protocols of the Institutional Animal Care and Use Committee of the Soochow University (IACUCSU).

\section{Cell proliferation assay}

Cell proliferation was detected by Cell Count Kit-8 (CCK-8; Dojindo Laboratories, Kumamoto, Japan). In brief, cells were plated in 96-well plates at a density of 1000 cells per well and cultured in growth medium. At specific points in time after plating $(0,7,24,48,72,96$, and $120 \mathrm{~h})$, the cell counts were determined according to the manufacturer's instructions.

For clonogenic survival assay, five hundreds cells were seeded into dishes $35 \mathrm{~mm}$ in diameter. After two weeks, the colonies formed were fixed with formaldehyde, stained with Giemsa, and counted using a colony counter soft at GBOX XR-5 (Gene Company Limited, HongKong, China). The plating efficiencies (PE) were determined using the following formula: $\mathrm{PE}(\%)$ = number of colonies formed / 500 plated cells $\times 100 \%$. All data points in figures represent three independent experiments.

\section{Soft agar colony formation assay}

Two thousand cells were suspended in $1 \mathrm{~mL}$ of $0.35 \%$ agarose in the growth medium, plated into $35 \mathrm{~mm}$ dishes with a bottom layer of $0.7 \%$ agarose. Cells were fed every 3 days with $1 \mathrm{ml}$ growth medium. Colonies $(>15 \mathrm{~mm})$ were counted two or three weeks after seeding. Data are presented as CFU (percent colony forming units in soft agar assay) $=$ mean of the number of colonies per dish/number of cells seeded per dish' $100 \%$. Data points in figures represent three independent experiments.

\section{In vivo tumorigenicity assays}

Ten female BALB/c nude mice (SPF, 4-6weeks, 18-20g) were bought from Experimental Animal Center for Soochow University, and they were housed under temperature-controlled conditions, underwent a reverse dark-light cycle, and provided with standard mouse pellets and tap-water ad libitum. The mice were randomly divided into three groups $(n=3)$ including $M-E$ group, $M-I$ group and $M-L$ group for injection MOSE cells. After animals were lightly anesthetized with isoflurane, $5 \times 10^{6}$ of cells were injected subcutaneously into the left and right flanks of each animal. Animals were palpated weekly for tumor formation. Tumor size was measured once per week and tumor volume was calculated using the formula, tumor volume $=0.5 \times$ length $\times$ width $^{2}$. Animals were killed as soon as tumor nodules reached a size of $1.2-1.5 \mathrm{~cm}$. The euthanasia/sacrifice of the mice was used by excessive $3 \%$ pentobarbital sodium ( 0.1 $\mathrm{ml} / 10 \mathrm{~g}$ ), following cervical dislocation to ensure the death of mice. This animal experiment was performed according to the protocols of the IACUCSU.

\section{PCR Array}

The total RNA was isolated from $10^{6}$ cells using TRIZOL (Life Technologies). cDNA was synthesized from $500 \mathrm{ng}$ of the total RNA using the $\mathrm{RT}^{2}$ first strand fit (SABiosciences, Frederick, MD). After all the control 
tests, the samples were analyzed using the mouse EMT RT ${ }^{2}$ profiler PCR array (PAMM-090Z, SABiosciences, Frederick, MD) that profiles the expression of 84 key genes. Altogether 84 different genes were simultaneously amplified in each sample. Five house-keeping genes (B2M, HPRT1, RPL13A, GAPDH, and ACTB), genomic DNA contamination control, reverse transcription control and positive PCR control were included in each PCR array. Briefly, the reaction mix was prepared from $2 \times$ SABiosciences $R^{2}{ }^{2} \mathrm{qPCR}$ master mix and $102 \mathrm{ml}$ of sample cDNA. Ten $\mathrm{ml}$ of this mixture was added into each well of the PCR array. PCR arrays were performed in 384-well plates on a 7500 real time PCR system (Applied Biosystem, Foster City, CA).

\section{Spheroid culture}

Sphere formation assay was performed using serum-free DMEM/F12 Medium, including $20 \mathrm{ng} / \mathrm{mL}$ $\mathrm{mEGF}, 20 \mathrm{ng} / \mathrm{mL}$ basic fibroblast growth factor, $100 \mathrm{U} / \mathrm{mL}$ penicillin and $100 \mu \mathrm{g} / \mathrm{mL}$ streptomycin, 2 $\mathrm{mg} / \mathrm{ml}$ insulin, $4 \mathrm{mg} / \mathrm{mL}$ heparin sodium, and $6 \mathrm{mg} / \mathrm{mL}$ glucose. Late MOSE cells were plated at a density of 1000 cells in $35 \mathrm{~mm}$ dish and cultured for 10 days. Round cell clusters larger than $100 \mathrm{~mm}$ were judged as spheres. Data was presented as sphere formation efficiency $(\%)=$ (number of spherical formation/plated cells $\times 100 \%$ ). Data points in figures represent three independent experiments.

\section{Limiting dilution assay}

Limiting dilution assay was performed to measure the number of cells required to generate at least 1 tumor sphere/well as previously described [29]. Briefly, serial two fold dilutions of M-I (from 0 to 5000 cells) and M-L cells (from 0 to 500 cells) were sorted into ultra-low 96-well plates with 6 wells per dilution, respectively. Cultures were fed $50 \mathrm{ml}$ of CSCs medium every 2 days until day 10 . Fraction of wells without spheres ( $y$-axis) was plotted against the number of cells plated per well (x-axis). Regression lines were plotted and $x$-intercept values calculated, which represent the number of cells required to form at least 1 tumor sphere in every well.

\section{Western-blotting}

Proteins were extracted with lysis buffer (Beyotime Biotechnology, Shanghai, China). The protein concentration of lysate was quantified using a bicinchonininc acid protein assay kit (Beyotime Biotechnology). Equal amounts of total proteins $(30 \mu \mathrm{g})$ were electrophoretically transferred onto a polyvinylidene fluoride membrane (Millipore, Boston, MA). After being blocked with $5 \%$ skimmed milk, the membranes were incubated with primary antibodies of pan-keratin, vimentin, E-cadherin, b-catenin, Slug, Snail, Nanog, and $\beta$-actin (Cell Signaling Technology, Danvers, MA) at a 1:1000 dilution. Secondary antibodies were purchased from Amersham Biosciences (Piscataway, $\mathrm{NJ}$ ) and the immune complexes were detected using an enhanced chemiluminescence (Millipore, Boston, MA) method according to the manufacturer's instructions.

\section{Immunofluorescences}


Cells were plated into micro-slides, and then fixed in $4 \%$ paraformaldehyde. Cells were permeabilized with $0.5 \%$ Triton followed by blocking with $5 \%$ BSA for at least 30 minutes at room temperature (RT). Incubations with primary antibodies were carried out for overnight at $4{ }^{\circ} \mathrm{C}$. Appropriate secondary antibodies conjugated to either AlexaFluor 488, AlexaFluor 543, or AlexaFluor 635 were carried out for 30 min at RT. Nuclear was counterstained with 4',6-diamidino-2-phenylindole (DAPI). Image was observed using SP5 confocal laser scan microscope (Leica, Wetxlar, Germany).

\section{Flow cytometry assay}

For stem cell marker analysis, $10^{6}$ cells were resuspended in PBS, incubated with fluorescenceconjugated monoclonal antibodies against CD44-FITC and CD117-PE (BD Pharmingen) at $4{ }^{\circ} \mathrm{C}$ for 10 min, and washed by PBS for 3 times. The samples were analyzed by FC500 flow cytometer (Beckman Coulter, Fullerton, CA).

\section{Histology and immunohistochemistry}

Resected ovaries and tumors were collected from mice, fixed in $4 \%$ paraformaldehyde. Routine paraffin embedding of tissues and hematoxylin and eosin staining of tissue sections were performed by histopathological laboratories of Soochow University (Suzhou, China). For immunohistochemistry, tissues were cut into $5 \mu \mathrm{m}$ sections. Slides were deparaffinized and rehydrated in an ethanol series. The sections were treated with $3 \% \mathrm{H}_{2} \mathrm{O}_{2} / \mathrm{MET}$ for 20 min and boiled with citrate buffer $(0.01 \mathrm{M}, \mathrm{pH} 6.00)$, then incubated overnight with indicated primary antibodies at $4^{\circ} \mathrm{C}$. After that, followed with PBS washed for 3 times, the slides were then incubated with secondary antibodies with HRP. The slides were incubated with DAB solution, and then counterstained with hematoxylin according to standard protocols.

\section{Statistical analysis}

Data were presented as mean \pm standard deviation (SD), and were analyzed with one way analysis of variance (ANOVA) and Student's t-test. $P<0.05$ were considered statistically significant.

\section{Results}

\section{The spontaneous neoplastic transformation of MOSE cells in vitro.}

It is well known that primary epithelial cells stop replication after $\sim 10$ passages in vitro. Owing to the specific physiological role of ovarian remodeling during ovulation, it is possible that OSE continues to proliferate when subcultured in vitro. We wondered whether establishing a cell model of spontaneous epithelial ovarian cancer represented distinct transitional states of neoplastic progression. We first isolated primary MOSE cells from mouse ovary and cultured them in vitro. The MOSE showed typical cobblestone-like phenotype and maintained contact inhibition of growth. The culture media used in the first 20 passages consisted of a 1:1 mixture of fresh and conditioned medium of existing cultures, which was different from previous report $[24,28]$ but proved to be crucial to maintain the cultures. The 
cultivation of these early, MOSE cells gradually entered the intermediate stage when cells displayed more spindle-shaped morphology and loss of cell-cell contact. MOSE cells finally converted into spindle-like mesenchymal morphology as evidenced by their multi-layered growths (Fig. 1A). Interestingly, MOSE cells were positive for both Pan-keratin and Vimentin during the long-term in vitro culture (Fig. 1B, C and D), suggesting that they had a dual characteristics of not only epithelial but mesenchymal nature as well.

To further elucidate these transitional stages, we determined the capacity of anchorage-independent growth, an in vitro hallmark of neoplastic transformation of cells. Both early ( $<20$ passages) and intermediate (21-80 passages) MOSE cells were unable to form colonies in soft agar. Notably, latepassage ( $>81$ ) MOSE cells were capable of forming $>30 \mathrm{~mm}$ colonies but at diminished efficiency compared with human ovarian cancer SKOV-3 cells (Fig. 2A). Compared with MOSE cells at earlier stages, late-passage MOSE cells exhibited dramatically increased plating efficiency and growth rate, another proliferative parameter that is often associated with neoplastic change (Fig. 2B and C). The average chromosomal number observed in early passages MOSE cells was 49 , and $36 \%$ of cells at this stage were hyperdiploid. But as the cells progressed, there was a trend toward increased numeric abnormalities. In the late-passage cells, the average chromosome number was 64 , with $43 \%$ were neartetraploid and hypertetraploid (Fig. 2D). Therefore, based on morphological change, chromosomal number and proliferating ability, we defined three sequential stages of transformed MOSE cells as M-E ( $\leq 20$ passages), $\mathrm{M}-\mathrm{I}$ (21-80 passages) and M-L ( $\geq 81$ passages) cells, respectively.

\section{Tumorigenicity of MOSE cells in vivo}

To assess oncogenic transformation of MOSE cells in vivo, we subcutaneously injected them into athymic nude mice. As expected, M-L cells formed tumors in three of three animals within four weeks post-injection. In contrast, M-E cells did not form any tumor by 12 weeks post-injection (Fig. 3A). Although $\mathrm{M}-\mathrm{I}$ cells formed smaller nodules with average volume of $17 \mathrm{~mm}^{3}$, HE staining showed only infiltrated lymphocytes (Fig. 3B and C). However, M-L cells developed relative fast growing tumors with an average volume of $1475.86 \mathrm{~mm}^{2}$, and showed malignant cells with a large nuclear to cytoplasmic ratio and neovascularization (Fig. 3). These tumor nodules showed high level of both Pan-keratin and Vimentin, indicating that they were similar in phenotype to that of epithelial ovarian cancer $[9,19,30]$ (Fig. 3D). These results showed that MOSE cells, over time, spontaneously develop characteristics of malignant cells and generate tumors consistent with epithelial ovarian cancer. Thus, progression of MOSE cells from a benign to a malignant phenotype in vitro may provide a model to dissect the progression of ovarian cancer.

\section{EMT alteration was accompanied with spontaneous oncogenic-transformation of MOSE cells}

EMT is believed to be an essential part of post-ovulatory remodeling process $[9,31]$. To determine whether the spontaneously transformed MOSE cells undergo EMT, we compared EMT-related gene expression profiling by using high-throughput EMT profiler ${ }^{T M}$ PCR array. Figure 4 shows that the expression of EMT-related genes, specifically Spp1, Wnt11, Cav2, Cald1, MSN, Jag1 and Vps13a, are 
markedly increased both in M-I and M-L cells. On the other hand, the level of mRNA down-regulated during EMT included Wnt5b, Ptp4a1, Mmp2, Mmp3, Col3a1, and Esr1. These genes were strongly reduced in malignant progression of MOSE cells (Fig. 4A, 4B and 4C). Furthermore, the mRNA levels of EMTinduced transcription factors (Snail, Slug, Tcf4 and Tgfb2) were significantly up-regulated, and those of epithelial markers (Krt19 and Krt7) were down-regulated in M-L cells (Fig. 4A). Indeed, the results of immunofluorescence studies also showed an increased expression of Snail and Slug in M-L cells, compared with M-E cells, as demonstrated by western-blotting analysis (Fig. 4D). Interestingly, level of Ecadherin and b-catenin in M-I cells increased dramatically (Fig. 4E). Collectively, as illustrated by the mesenchymal morphology of M-L cells shown in Figure $1 \mathrm{~A}$, these results indicated that the neoplastic transformation of MOSE was accompanied by continuous EMT-inducing signals including notable increase of Snail and Slug.

\section{Stem-cell-like properties were correlated with neoplastic transformation of MOSE cells}

There is recent evidence that OSE at the junction area contains a cancer-prone stem cell niche [10, 11, 31]. We wondered whether the neoplastic transformation of normal surface epithelial cells correlates with acquisition of stemness. So, the stem cell-like properties of MOSE cells were monitored during their longterm culture in vitro. The percentages of $\mathrm{CD} 44^{+}$and $\mathrm{CD} 117^{+}$, markers for ovarian cancer stem cells, were found to be up-regulated post neoplastic transformation of MOSE cells (Fig. 5A). Consistently, mRNA of stem-related genes, such as Nanog and Sox2, gradually increased and KLF4 decreased during malignant progression of MOSE cells (Fig. 5B). Interestingly, the M-L cells displayed obviously self-renewal properties, which formed larger and more spheres from a single cell suspension under serum-free medium. The sphere forming efficiency (SFE) was markedly higher in M-L cells, compared with that in M$\mathrm{E}$ and $\mathrm{M}-\mathrm{I}$ cells (Fig. 5C). These findings suggested that stem-like cells in ovarian surface epithelium might be involved in the progression of spontaneous neoplastic transformation of MOSE cells in vitro.

A single tumor sphere resulting from the proliferation of a single cancer stem cell coupled with limiting dilution analysis allows for the determination of the minimal frequency of repopulating tumor sphere cells within the cell population. Figure 5D revealed that at least $1072 \mathrm{M}-\mathrm{I}$ cells were required to generate one tumor sphere, whereas $431 \mathrm{M}-\mathrm{L}$ cells were required. M-L cells formed tumors in nude mice within 4 weeks after $10^{6}$ cells were subcutaneously injected. However, sphere forming cells produced tumors at 5 of 5 sites with $10^{5}$ cells at each site within two weeks. Furthermore, expression of CD44 based on immunofluorescence staining was higher in tumor tissue injected by sphere forming cells than that of $\mathrm{M}$ $L$ cells (Fig. 5E). Taken together, these results further suggest that stem-like acquisition of MOSE cells are closely correlated with the development of ovarian cancer.

\section{Discussion}

In this study, we report, for the first time, that both EMT alteration and stemness acquisition are closely correlated with the spontaneous neoplastic transformation of MOSE cells during long-term culture in vitro. Primary MOSE cells maintained proliferation and underwent spontaneous neoplastic 
transformation when subcultured for more than 80 passages in vitro, an observation consistent with several earlier reports $[24,26-28]$.

It is widely accepted that transformation process often involves alteration of oncogenes and/or tumor suppressor gene. Several experimental models have been developed to study the transformation of genetically modified OSE, including K-ras [22], KLF8 [20], Akt1[32], p53 and Rb1 [33]. Although these genetic models provide important insights into ovarian tumor biology, they are not representative of the heterogeneous and spontaneous nature of EOC. It is unclear whether alteration of EMT, physiologic function of normal ovarian surface epithelial, may be conducive to OSE transformation.

The single-layered epithelial cell structure of human OSE belies its complex biology. Due to its active role in both follicular rupture and subsequent ovarian remodeling, OSE possesses extraordinary cellular proliferative capacity in vitro, which often promotes the transformation of these epithelial cells $[9,19]$. The capacity of OSE to undergo EMT in response to postovulatory stimuli has been proposed to confer advantage to the postovulatory repair of the OSE. Hence, EMT is a part of normal OSE physiology and failure to undergo such process may be one of the reasons of the initiation and metastasis of ovarian cancer. The fact that OSE can transit back and forth between epithelial and mesenchymal phenotypes is believed to be part of the normal process of post-ovulatory ovarian remodeling [9, 31]. We also found that primary MOSE cells showed a dual nature of not only as epithelial but also as mesenchymal cells, and demonstrated that spontaneously malignant transformation progressed with a concomitant increase in EMT-transcription factor Snail and Slug. Additionally, the normal OSE is not only a somatic stem cell source given its role in repeated ovulation, but also a potential progenitor of EOC. Cancer-prone stem cell niche was identified on the OSE [11]. Consequently, whether stem-cell properties and EMT alteration are involved in malignant transformation of OSE remains incompletely understood. After the models established by Robe and Pauc et al. [24, 28], we have again identified that mouse OSE cells can spontaneously undergo neoplastic transformation during in vitro culturing. In our model, three transitional stages including early, immediately and late, were distinguished by morphologic phenotypes, the alteration of both EMT and stemness, proliferation in vitro and in vivo.

M-E cells represent the earliest transitional state of our model, characterized by typical cobblestoneepithelial appearance, a slow growth rate, and inability to anchorage-independent growth. They favor strong cell-substratum as well as cell-cell contact. Interestingly, in this stage, the stem and/or progenitor cell markers, such as CD44 and CD117 were often detected, even though in low proportion. The existence of somatic stem cells is likely to reside in distinct tissue compartments of the female reproductive system, because of the control for menstrual cycle [17]. Indeed, it has been well established that somatic stem cells exists in the OSE due to the cyclical ruptured and regeneration that occurs with ovulation [31]. These stem cells are characterized by a high capacity of self-renewal and slow cycling in physiological conditions, but they exhibit high proliferative potential during wound healing and in tissue culture. Normal label-retaining OSE cells were demonstrated to exhibit stem/progenitor cell characteristics [10]. Stem cell niche of OSE is verified to locate in the hilum region of the ovary [11]. In addition, we find that M-E cells are able to maintain growth only in a 1:1 mixture of fresh and conditioned medium. The extracellular 
proteins, such as Mmp2, Mmp5, Col3a1, and Wnt5b, were increased in M-E cells, suggesting conditioned medium containing extracellular proteins could promote cell proliferation. Hence, further studies on metabolomics profiling of conditioned medium may help to identify key components of early preneoplastic ovarian cancer.

With increased passage numbers, proliferative phenotype is paralleled with the neoplastic ability of M-I cells. But they are only identified as atypical hyperplasia. These cells recapitulate the continuum of cellular changes that represent the transition at early passage from mild to moderate at intermediate passage. The proportion of tetraploid cells increased from $10 \%$ at early to $30 \%$ at intermediate stage. Cells undergoing EMT are known to lose E-cadherin and gain Vimentin expression, resulting in tumor cell invasion and metastasis [34]. But Burkhalter et al found that E-cadherin expression is increased with malignant transformation in ovarian cancer [35]. The expression of E-cadherin and Pan-keratin, even bcatenin in the intermediate stage is the highest in the present study. In accordance with other reports [19, 24], we also found that MOSE cells exhibited both mesenchymal and epithelial markers continuously. This mixed phenotype enables the OSE cells to respond rapidly to a variety of environmental, hormonal, and stress factors, but it is also thought to contribute to the onset of neoplastic transformation by rendering the cells more susceptible to transition from an epithelial to a mesenchymal-like phenotype.

M-L cells represent the most aggressive transitional stage and display features similar to tumor cells, such as increased proliferation, and above $50 \%$ triploid and tetraploid cells. M-L cells are oncogenic transformed supported by two key evidences, enhanced capacity for anchorage-independent growth in vitro and rapid tumorigenic potential in vivo. These characteristics are associated with a continued expression of Pan-keratin, Vimentin and b-catenin and up-regulation of EMT-inducing transcription factor Snail and Slug. As we all know, EMT is a highly conserved cellular program that allows polarized, welldifferentiated epithelial cells to convert to depolarized, motile mesenchymal cells. Moreover, the transient induction of EMT in large populations of cancer cells may make it possible that the generation of relatively unlimited numbers of CSCs $[15,36]$. The stem cells of certain epithelial organs show many of the attributes of the mesenchymal cell state. Therefore, M-I cells displayed phenotype of both EMT alteration and stemness acquisition.

There are several characteristics of EOC which indicate that it may be a stem cell-driven disease. Such as, EOC can generate differentiated subtypes that recapitulate the histology of other normal gynecologic tissues and the high rate of chemoresistance and recurrence after successful initial treatment. In clinical and experimental studies, the high level of stem-cell markers, such as Nanog, ALDH-1, CD44, CD117, CD133 and Sox-2, were found to be associated with poor outcomes of EOC patients [30, 37-40]. CSCs contribute to the aggressive behavior of EOC $[38,41]$. Our findings also suggest that acquisition of stemness is associated with malignant spontaneous transformation of OSE cells in long-term culture.

\section{Conclusions}


In conclusion, we established a model representing early, intermediate, and late stages of neoplastic transformation of ovarian surface epithelium. Alteration of EMT and acquisition of stemness are closely correlated to spontaneously oncogenic transformation of OSE cells. The model is a powerful tool for in vitro mechanistic studies, as well as for efficacy studies in vivo of both chemotherapeutic treatment regimens and chemopreventive strategies.

\section{Abbreviations}

CSCs: cancer stem-like cells; EMT: epithelial-mesenchymal transition; MOSE cells: mouse ovary surface epithelial cells; M-E: early stage of MOSE cells; M-I: intermediated stage of MOSE cells; M-L: late stage of MOSE cells.

\section{Declarations}

\section{Ethics approval and consent to participate}

The performance of the animal experiments followed the 3R principle, in order to minimize the number of animals recruited for the experiments and to reduce their burden. All animal experiment was performed according to the protocols of IACUCSU. The approval number is ECSU-201800049.

\section{Consent for publication}

Not applicable.

\section{Availability of data and materials}

The datasets used and/or analyzed during the current study are available from the corresponding author on reasonable request.

\section{Competing interests}

The authors declare that they have no competing interests.

\section{Funding}

This study was supported by the National Natural Scientific Funding of China (grant No. 81872622, 81673151, U1832140, and 81372979), and BL is the Principle Investigator. The funding source had no role in the study design, data collection and analysis, the decision to publish, or the preparation of this manuscript.

\section{Authors' contributions}

BL conceived and designed the experiments; $H Z, Y G, L L, Y H$ and $P W$ performed the experiments; $H Z$ and YG analyzed the data; FJ and ZZ contributed material and analysis tools; BL, ZZ and TOM K.HEI drafted 
and revised the paper. All authors read and approved the manuscript

Acknowledgments

Not applicable.

\section{References}

1. Siegel R, Naishadham D, Jemal A: Cancer statistics, 2013. CA: a cancer journal for clinicians 2013, 63(1):11-30.

2. Bast RC, Jr., Hennessy B, Mills GB: The biology of ovarian cancer: new opportunities for translation. Nature reviews Cancer 2009, 9(6):415-428.

3. Kurman RJ, le-Ming S: Pathogenesis of ovarian cancer: lessons from morphology and molecular biology and their clinical implications. International Journal of Gynecological Pathology Official Journal of the International Society of Gynecological Pathologists 2008, 27(2):151.

4. Wright JW, Pejovic T, Fanton J, Stouffer RL: Induction of proliferation in the primate ovarian surface epithelium in vivo. Human reproduction 2008, 23(1):129-138.

5. Dubeau L: The cell of origin of ovarian epithelial tumours. The Lancet Oncology 2008, 9(12):11911197.

6. Bowtell DD: The genesis and evolution of high-grade serous ovarian cancer. Nature reviews Cancer 2010, 10(11):803-808.

7. Lengyel E: Ovarian cancer development and metastasis. The American journal of pathology 2010, 177(3):1053-1064.

8. Kurman RJ, Shih le M: The origin and pathogenesis of epithelial ovarian cancer: a proposed unifying theory. The American journal of surgical pathology 2010, 34(3):433-443.

9. Ahmed N, Thompson EW, Quinn MA: Epithelial-mesenchymal interconversions in normal ovarian surface epithelium and ovarian carcinomas: an exception to the norm. Journal of Cellular Physiology 2007, 213(3):581-588.

10. Szotek PP, Chang HL, Brennand K, Fujino A, Pieretti-Vanmarcke R, Lo Celso C, Dombkowski D, Preffer F, Cohen KS, Teixeira J et al: Normal ovarian surface epithelial label-retaining cells exhibit stem/progenitor cell characteristics. Proceedings of the National Academy of Sciences of the United States of America 2008, 105(34):12469-12473.

11. Flesken-Nikitin A, Hwang Cl, Cheng CY, Michurina TV, Enikolopov G, Nikitin AY: Ovarian surface epithelium at the junction area contains a cancer-prone stem cell niche. Nature 2013, 495(7440):241245.

12. Steg AD, Bevis KS, Katre AA, Ziebarth A, Dobbin ZC, Alvarez RD, Zhang K, Conner M, Landen CN: Stem cell pathways contribute to clinical chemoresistance in ovarian cancer. Clinical cancer research 2012, 18(3):869-881. 
13. Marchini S, Fruscio R, Clivio L, Beltrame L, Porcu L, Nerini IF, Cavalieri D, Chiorino G, Cattoretti G, Mangioni $\mathrm{C}$ et al: Resistance to platinum-based chemotherapy is associated with epithelial to mesenchymal transition in epithelial ovarian cancer. Eur J Cancer 2013, 49(2):520-530.

14. Nuti SV, Mor G, Li P, Yin G: TWIST and ovarian cancer stem cells: implications for chemoresistance and metastasis. Oncotarget 2014, 5(17):7260-7271.

15. Mani SA, Guo W, Liao MJ, Eaton EN, Ayyanan A, Zhou AY, Brooks M, Reinhard F, Zhang CC, Shipitsin $\mathrm{M}$ et al: The epithelial-mesenchymal transition generates cells with properties of stem cells. Cell 2008, 133(4):704-715.

16. Lili LN, Matyunina LV, Walker LD, Wells SL, Benigno BB, McDonald JF: Molecular profiling supports the role of epithelial-to-mesenchymal transition (EMT) in ovarian cancer metastasis. Journal of ovarian research 2013, 6(1):49.

17. Lopez J, Valdez-Morales FJ, Benitez-Bribiesca L, Cerbon M, Carranca AG: Normal and cancer stem cells of the human female reproductive system. Reproductive biology and endocrinology 2013, 11:53.

18. Bowen NJ, Walker DE, Matyunina LV, Logani S, Totten KA, Benigno BB, Mcdonald JF: Gene expression profiling supports the hypothesis that human ovarian surface epithelia are multipotent and capable of serving as ovarian cancer initiating cells. BMC Medical Genomics 2009, 2(1):1-14.

19. Auersperg N, Wong AS, Choi KC, Kang SK, Leung PC: Ovarian surface epithelium: biology, endocrinology, and pathology. Endocrine reviews 2001, 22(2):255-288.

20. Lu H, Wang X, Urvalek AM, Li T, Xie H, Yu L, Zhao J: Transformation of human ovarian surface epithelial cells by Kruppel-like factor 8. Oncogene 2014, 33(1):10-18.

21. Yang G, Thompson JA, Fang B, Liu J: Silencing of H-ras gene expression by retrovirus-mediated siRNA decreases transformation efficiency and tumorgrowth in a model of human ovarian cancer. Oncogene 2003, 22(36):5694-5701.

22. Mullany LK, Fan HY, Liu Z, White LD, Marshall A, Gunaratne P, Anderson ML, Creighton CJ, Xin L, Deavers $\mathrm{M}$ et al: Molecular and functional characteristics of ovarian surface epithelial cells transformed by KrasG12D and loss of Pten in a mouse model in vivo. Oncogene 2011, 30(32):35223536.

23. Liu J, Yang G, Thompson-Lanza JA, Glassman A, Hayes K, Patterson A, Marquez RT, Auersperg N, Yu Y, Hahn WC et al: A genetically defined model for human ovarian cancer. Cancer research 2004, 64(5):1655-1663.

24. Roberts PC, Mottillo EP, Baxa AC, Heng HH, Doyon-Reale N, Gregoire L, Lancaster WD, Rabah R, Schmelz EM: Sequential molecular and cellular events during neoplastic progression: a mouse syngeneic ovarian cancer model. Neoplasia 2005, 7(10):944-956.

25. Roberts PC, Schmelz EM: In vitro model of spontaneous mouse OSE transformation. Methods in molecular biology 2013, 1049:393-408.

26. McCloskey CW, Goldberg RL, Carter LE, Gamwell LF, Al-Hujaily EM, Collins O, Macdonald EA, Garson $\mathrm{K}$, Daneshmand $\mathrm{M}$, Carmona $\mathrm{E}$ et al: A new spontaneously transformed syngeneic model of high- 
grade serous ovarian cancer with a tumor-initiating cell population. Frontiers in oncology 2014, 4:53.

27. Lucie Gregoire RR, Eva-Maria Schmelz, Adnan Munkarah, Paul C. Roberts and Wayne D Lancaster: Spontaneous Malignant Transformation of Human Ovarian Surface Epithelial Cells in vitro. Clinical Cancer Research 2001, 7(11):4280-4287

28. Roby KF, Taylor CC, Sweetwood JP, Cheng Y, Pace JL, Tawfik O, Persons DL, Smith PG, Terranova PF: Development of a syngeneic mouse model for events related to ovarian cancer. Carcinogenesis 2000, 21(4):585-591.

29. Singh SK, Clarke ID, Terasaki M, Bonn VE, Hawkins C, Squire J, Dirks PB: Identification of a Cancer Stem Cell in Human Brain Tumors. Cancer research 2003, 63(18):5821-5828

30. Liu M, Mor G, Cheng H, Xiang X, Hui P, Rutherford T, Yin G, Rimm DL, Holmberg J, Alvero A et al: High Frequency of Putative Ovarian Cancer Stem Cells With CD44/CK19 Coexpression Is Associated With Decreased Progression-Free Intervals In Patients With Recurrent Epithelial Ovarian Cancer. Reprod Sci 2013, 20(5):605-615.

31. Chang HL, Maclaughlin DT, Cambridge SB: Somatic stem cells of the ovary and their relationship to human ovarian cancers. Stembook 2008.

32. King SM, Quartuccio SM, Vanderhyden BC, Burdette JE: Early transformative changes in normal ovarian surface epithelium induced by oxidative stress require Akt upregulation, DNA damage and epithelial-stromal interaction. Carcinogenesis 2013, 34(5):1125-1133.

33. Fleskennikitin A, Choi KC, Eng JP, Shmidt EN, Nikitin AY: Induction of Carcinogenesis by Concurrent Inactivation of p53 and Rb1 in the Mouse Ovarian Surface Epithelium. Cancer research 2003, 63(13):3459-3463

34. Christiansen JJ, Rajasekaran AK: Reassessing epithelial to mesenchymal transition as a prerequisite for carcinoma invasion and metastasis. Cancer research 2006, 66(17):8319-8326.

35. Burkhalter RJ, Symowicz J, Hudson LG, Gottardi CJ, Stack MS: Integrin regulation of beta-catenin signaling in ovarian carcinoma. The Journal of biological chemistry 2011, 286(26):23467-23475.

36. Lv L, Zhang T, Yi Q, Huang Y, Wang Z, Hou H, Zhang H, Zheng W, Hao Q, Guo Z et al: Tetraploid cells from cytokinesis failure induce aneuploidy and spontaneous transformation of mouse ovarian surface epithelial cells. Cell cycle 2012, 11(15):2864-2875.

37. Ffrench B, Gasch C, O'Leary JJ, Gallagher MF: Developing ovarian cancer stem cell models: laying the pipeline from discovery to clinical intervention. Molecular cancer 2014, 13:262.

38. Bapat SA, Mali AM, Koppikar CB, Kurrey NK: Stem and progenitor-like cells contribute to the aggressive behavior of human epithelial ovarian cancer. Cancer research 2005, 65(8):3025-3029.

39. Zanotti L, Romani C, Tassone L, Todeschini P, Tassi RA, Bandiera E, Damia G, Ricci F, Ardighieri L, Calza $S$ et al: MAL gene overexpression as a marker of high-grade serous ovarian carcinoma stemlike cells that predicts chemoresistance and poor prognosis. Bmc Cancer 2017, 17.

40. Luo L, Zeng J, Liang B, Zhao Z, Sun L, Cao D, Yang J, Shen K: Ovarian cancer cells with the CD117 phenotype are highly tumorigenic and are related to chemotherapy outcome. Experimental and molecular pathology 2011, 91(2):596-602. 
41. Zhang S, Balch C, Chan MW, Lai HC, Matei D, Schilder JM, Yan PS, Huang TH, Nephew KP: Identification and characterization of ovarian cancer-initiating cells from primary human tumors. Cancer research 2008, 68(11):4311-4320.

42. Shibue T, Weinberg RA: EMT, CSCs, and drug resistance: the mechanistic link and clinical implications. Nature reviews Clinical oncology 2017, 14(10):611-629.

\section{Figures}

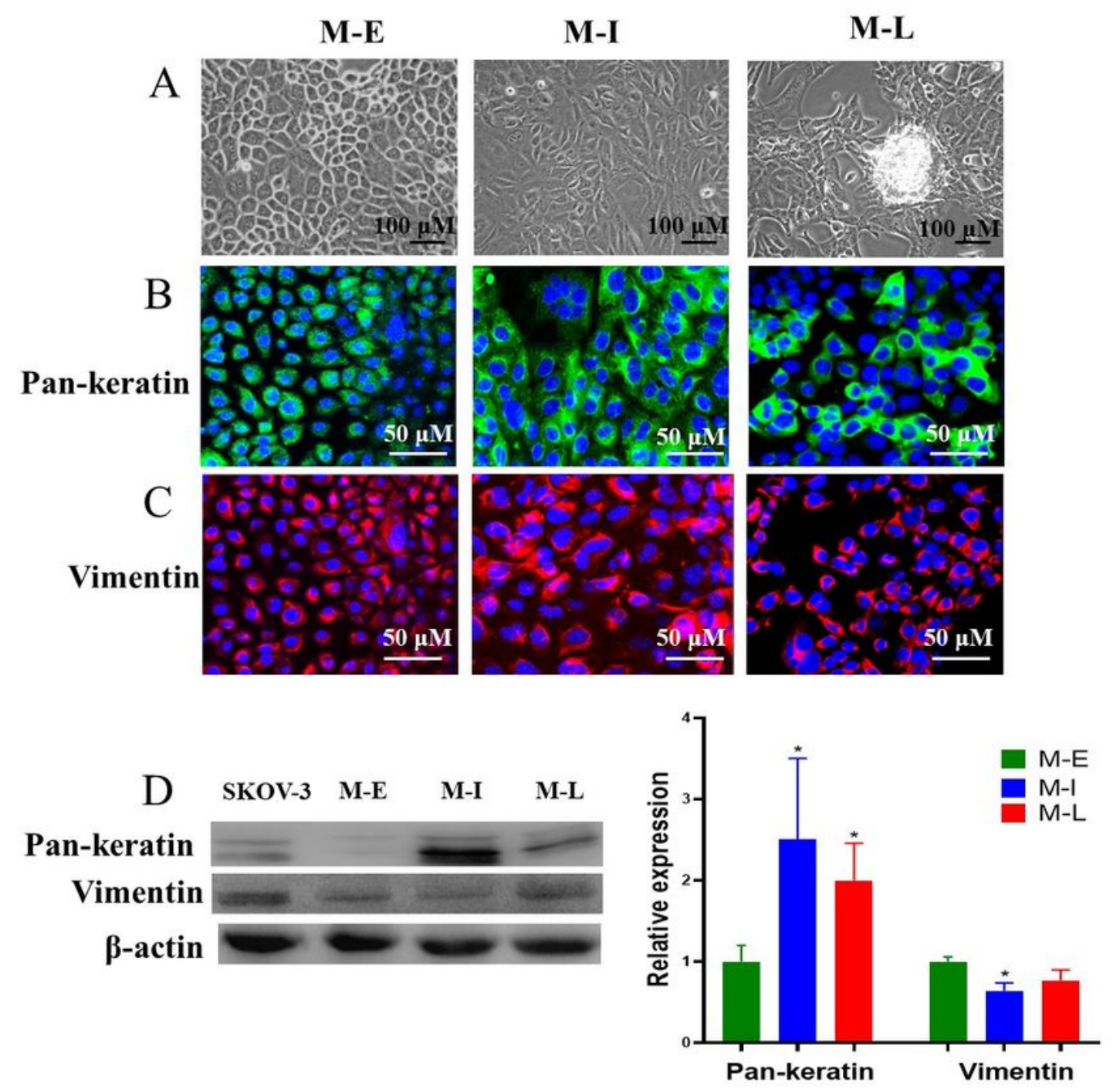

Figure 1

MOSE cells exhibit positive marker of both epithelial and mesenchymal during spontaneously malignant transformation. (A) Morphology of MOSE cells at different transitional stage in vitro. Early, intermediate and late passage MOSE cells were presented as M-E, M-I, and M-L, respectively. (B) Representative 
images of MOSE cells staining with Pan-keratin (green) and DAPI (blue). (C) Representative images of MOSE cells staining with Vimentin (red) and DAPI (blue). (D) Western blotting analysis of Pan-keratin and Vimentin in $M-E, M-I$ and $M-L$ cells. $\beta$-actin served as a loading control. Error bars represent SEM and statistical analysis was performed using Student's test. * $P<0.05$ compared with M-E cells.
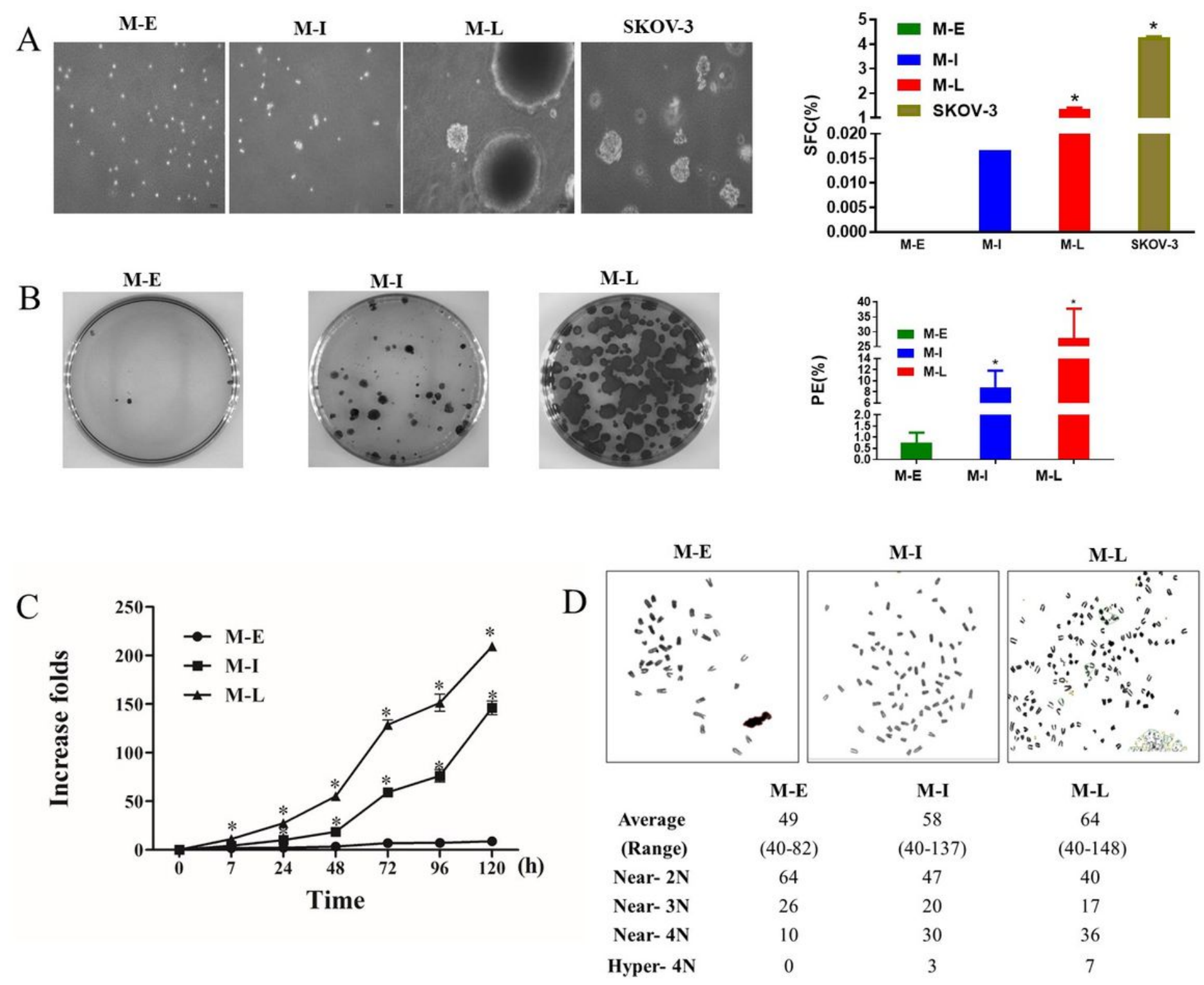

Figure 2

Proliferation ability of MOSE cells was dramatically increased with malignant transformation in vitro. (A) Colony formation in soft agar. Left: Representative images of colonies in soft agar. Right: Relative percentage of colony forming units. SKOV-3 cells were used as positive control. (B) Colony formation in plate. Left: Representative images of colonies in plate. Right: Relative plating colony efficiencies. (C) Cell proliferation rate at different transitional stage by CCK8 assay. (D) Upper: Representative karyotype of MOSE cells at different transitional stage ( $(630)$. Lower: Summary of chromosomal number analysis of 
MOSE transitional stages. Error bars represent SEM and statistical analysis was performed using Student's test. ${ }^{*} p<0.05$ compared with M-E cells.
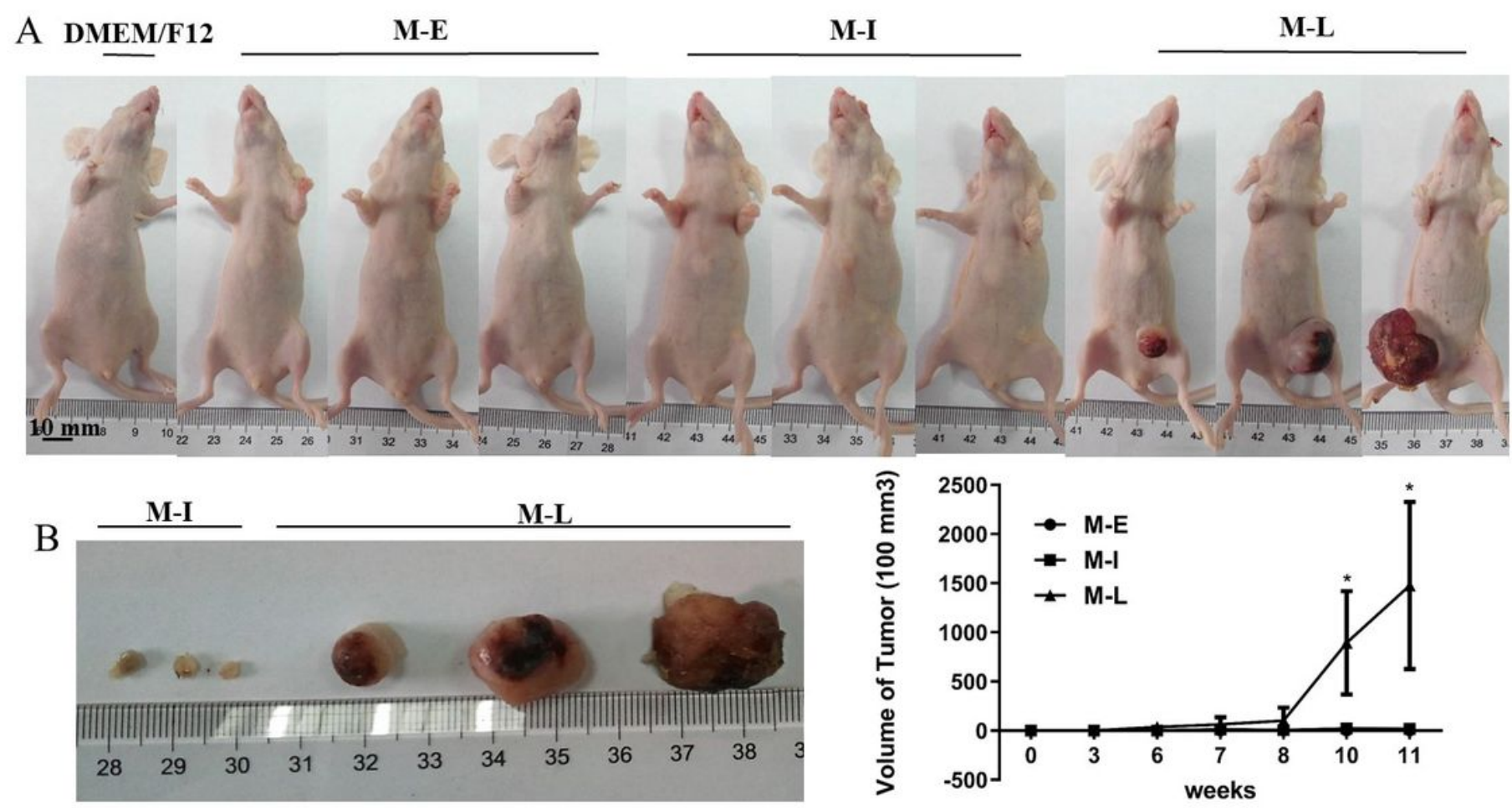

$\mathrm{C}$

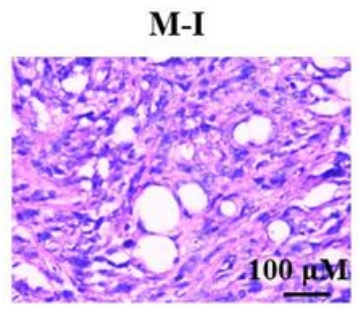

M-L

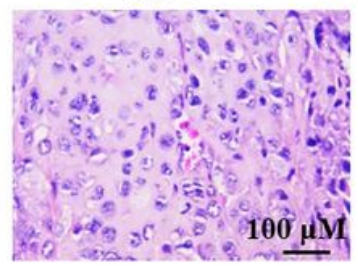

$\mathrm{D}$

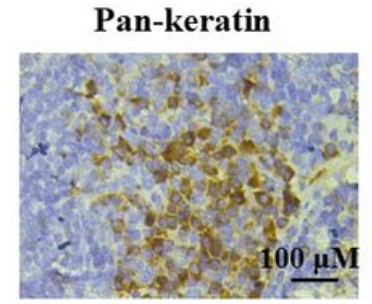

Vimentin

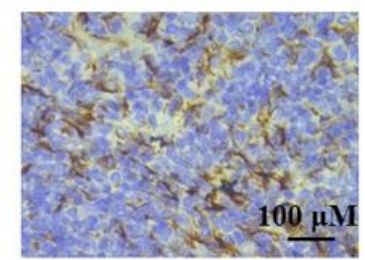

\section{Figure 3}

Tumorigenicity of MOSE cells in vivo. (A) Representative subcutaneous tumors formed by the indicated cells. DMEM/F12 was used as negative controls. (B) Tumor nodules were taken the time of euthanasia (left), and tumor volumes were recorded at the indicated time points (right). Error bars represent SEM and statistical analysis was performed using Student's test. * $\mathrm{p}<0.05$ compared with M-I cells. (C) HE staining of tumor generated from M-I and M-L cells. (D) Immunohistochemistry staining of epithelial markers Pankeratin (left) and mesenchymal markers Vimentin (right). 
A $\quad$ B

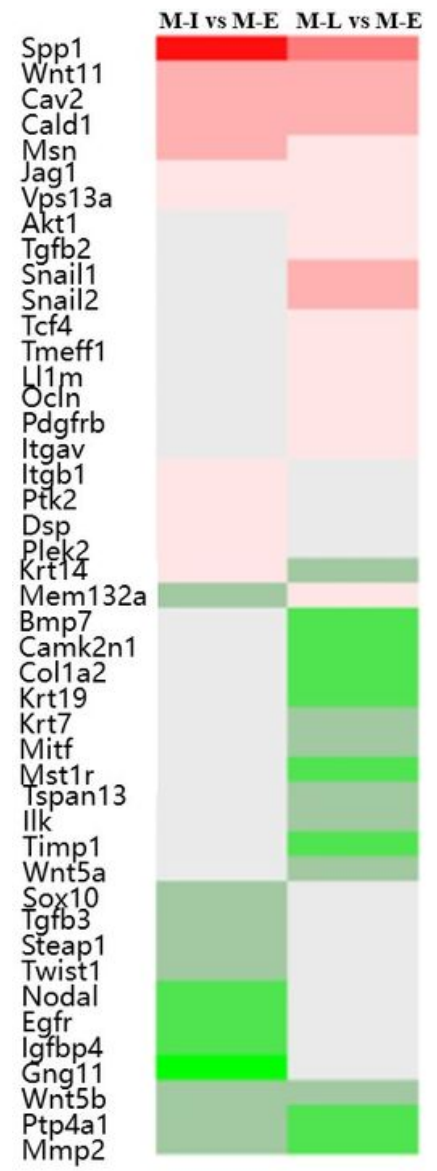

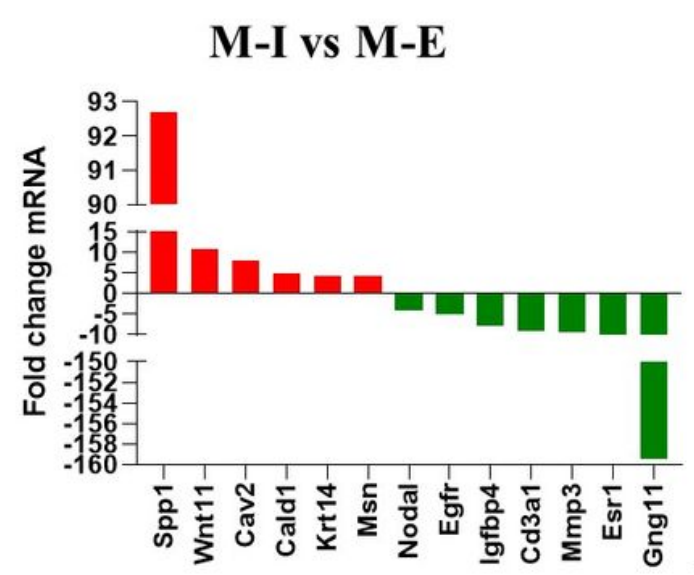

$\mathrm{D}$
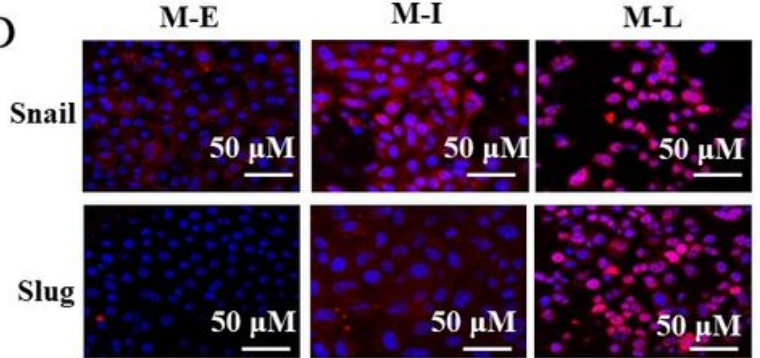

C E
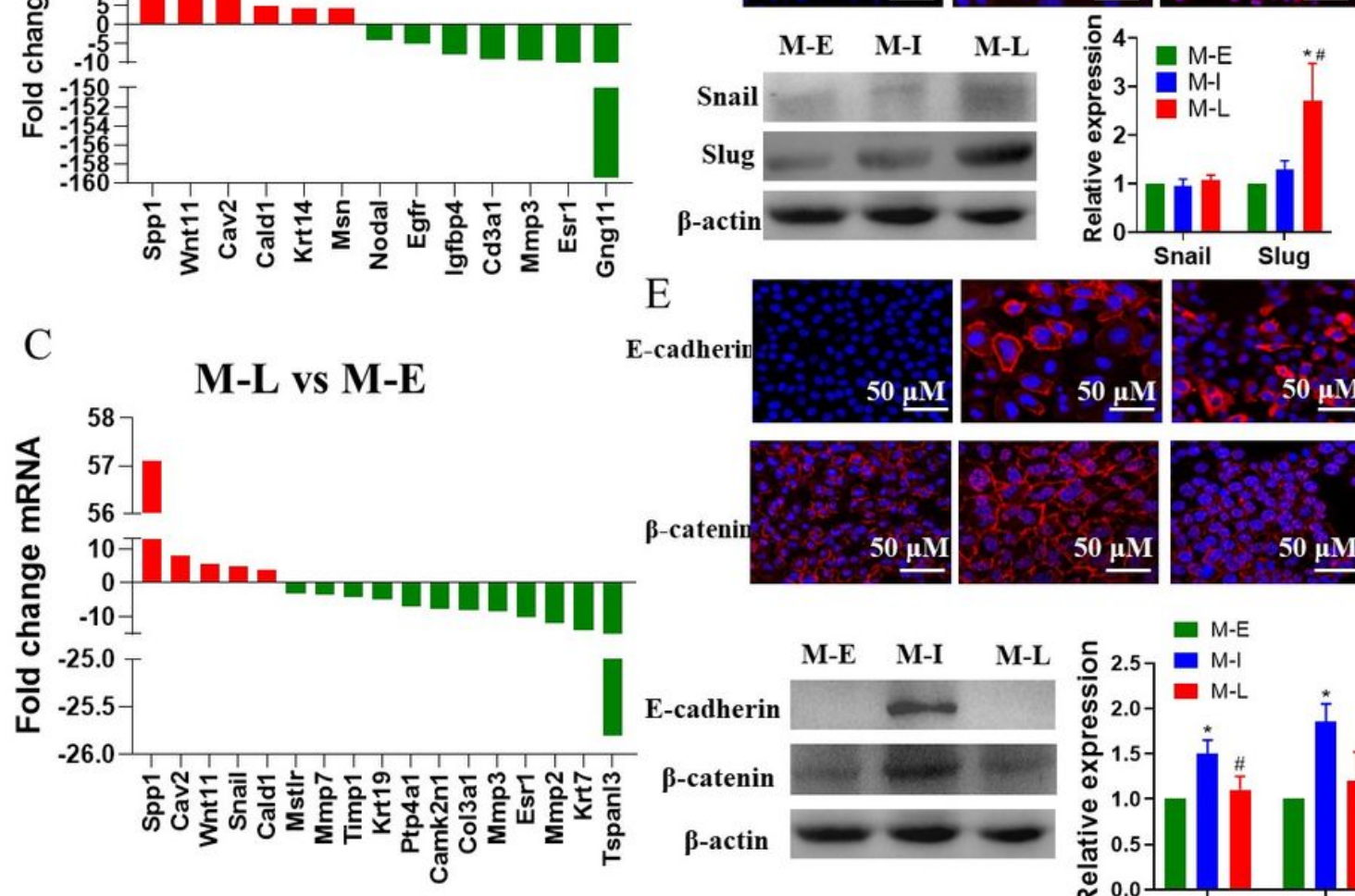

E-cadherir
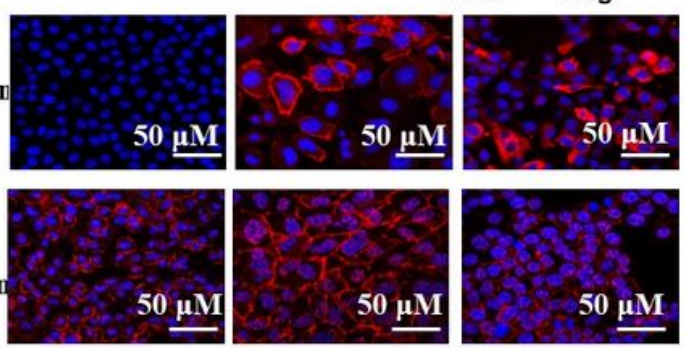

及-catenin

$50 \underline{\mu \mathrm{M}}$

$50 \mu \mathrm{M}$

$50 \underline{\mu \mathrm{M}}$

M-E M-I

E-cadherin
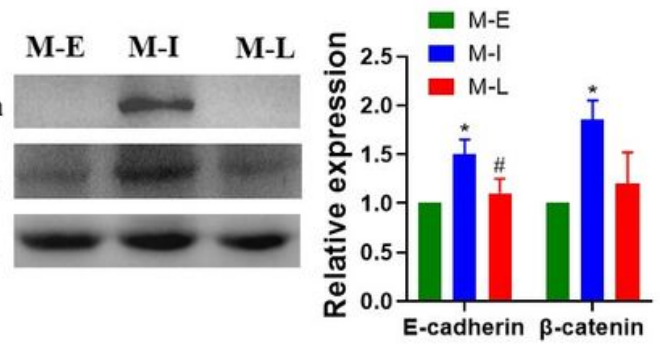

\section{Figure 4}

The alteration of EMT contributes to oncogenic transformation of MOSE cells. (A) Differential genes that were up-regulated (Red) or down-regulated (Green) by more than 3-fold. (B) Histograms depict the fold change differences in expression between $M-I$ and $M-E$. (C) Histograms depict the fold change differences in expression between $M-L$ and $M-E$. (D) Immunofluorescence staining and western blotting analysis of Snail and Slug of MOSE cells at different transitional stage. (E) Immunofluorescence staining and

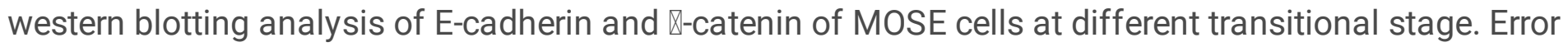
bars represent SEM and statistical analysis was performed using Student's test. * $p<0.05$ compared with M-E cells; \# $p<0.05$ compared with M-I cells. 

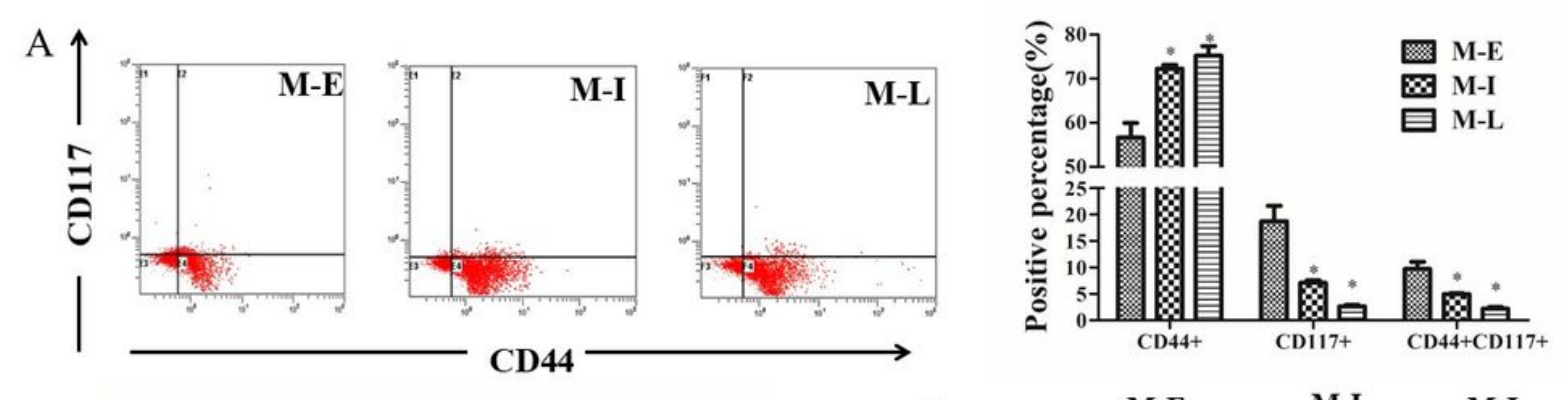

B

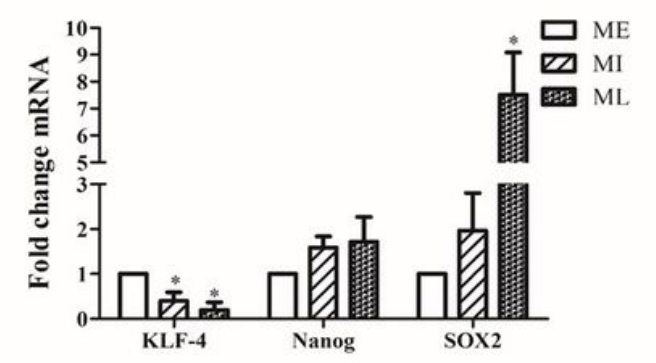

$\mathrm{D}$

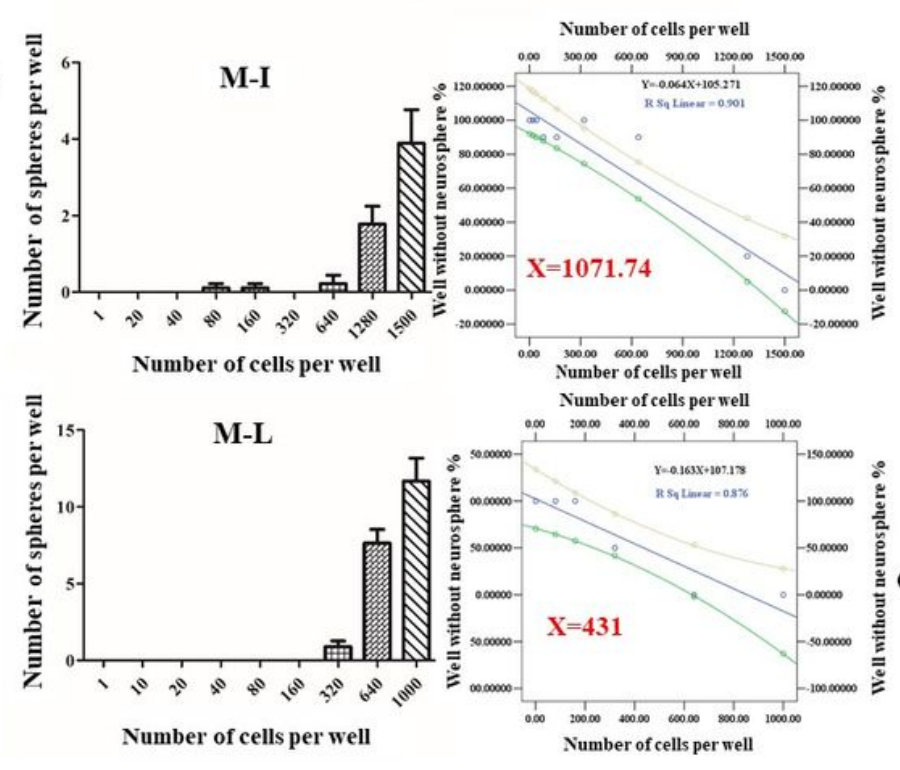

$\mathrm{C}$
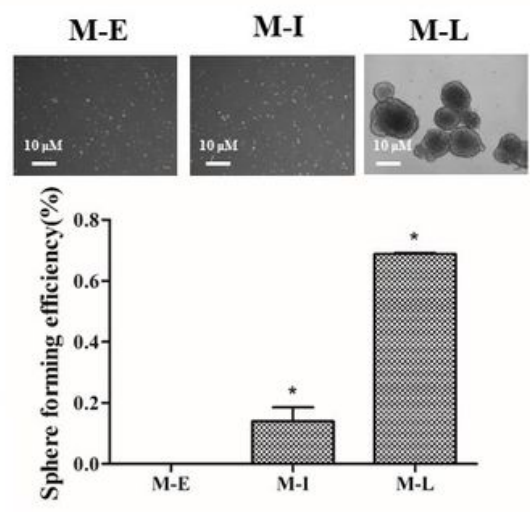

E

M-L

SFCs
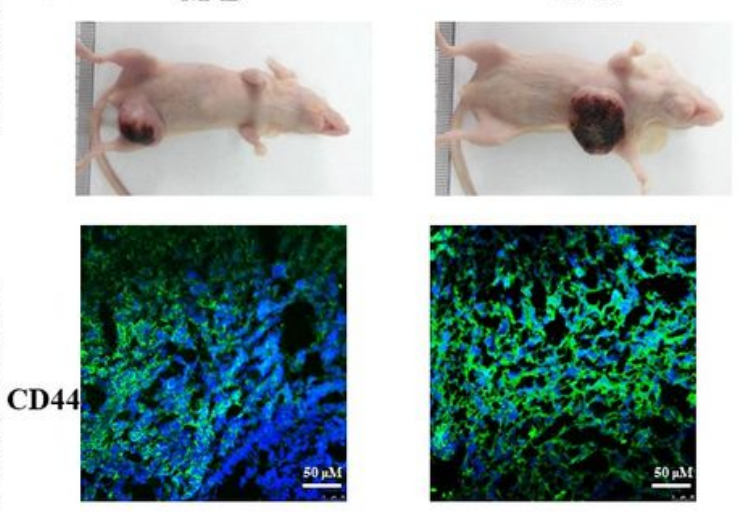

Figure 5

\section{Figure 5}

The acquisition of stemness involves in oncogenic transformation of MOSE cells. (A) Left: Flow cytometry analysis on the expression of CD44+/CD117+ stem cell markers. Right: The percentage of CD44+, CD117+ and CD44+/CD117+ cells from M-E, M-I and M-L cells (right). (B) Quantitative RT-PCR analysis of stemness-related genes expression in $\mathrm{M}-\mathrm{E}, \mathrm{M}-\mathrm{I}$ and $\mathrm{M}-\mathrm{L}$ cells. The expression of each gene was normalized to GAPDH. (C) Representative pictures (upper) and quantification (lower, means \pm SD) of sphere-forming cells (SFCs) in M-E, M-I and M-L. (D) Limiting dilution assay showed that M-L cells possessed higher capability of sphere formation than M-I cells. (E) Upper: Representative subcutaneous tumors formed by M-L and SFCs. Lower: Immunofluorescence staining of CD44 in M-L and SFCs generated tumors. Error bars represent SEM and statistical analysis was performed using Student's test. *P $<0.05$ compared with M-E cells; \# $\mathrm{p}<0.05$ compared with $\mathrm{M}-\mathrm{I}$ cells. 


\section{Supplementary Files}

This is a list of supplementary files associated with this preprint. Click to download.

- NC3RsARRIVEGuidelinesChecklistBCAND1903008.docx 